\title{
Translation and Validation of a Health-Related Quality of Life Instrument for 18-60-Month-Old Children in Korea
}

\author{
Kyung-Sook Bang ${ }^{1}$, Sung-Hee Park ${ }^{2}$, Hyun-Ju Kang ${ }^{3}$ \\ ${ }^{1}$ College of Nursing and the Research Institute of Nursing Science, Seoul National University, Seoul; ${ }^{2}$ Department of Nursing, Kunsan National University, \\ Gunsan; ${ }^{3}$ Department of Nursing, Bucheon University, Bucheon, Korea
}

Purpose: The purpose of this study was to validate the Korean version of the TNO-AZL Preschool Children Quality of Life (TAPQOL) questionnaire for children aged 18-60 months. Methods: The participants were 223 mothers of healthy children from nurseries and kindergartens, and 54 mothers of hospitalized children from a university children's hospital in Korea. Data were collected in 2011. Principal component factor analysis and known-groups method were used to confirm construct validity, and internal consistency was used to determine reliability. Results: According to the factor analysis, 11 factors with an eigenvalue over 1 were extracted, and these factors explained $73.5 \%$ of the variance. All dimensions except two had Cronbach's alpha coefficients greater than .7. The mean TAPQOL scores of the hospitalized group were significantly lower than those of the healthy group $(p=.001)$ for all four functions (physical, emotional, social, and cognitive). Conclusion: The TAPQOL showed acceptable validity and reliability, and can be used to measure children's health-related quality of life in Korea, and can be considered for use in other Asian countries.

Key words: Quality of life, Translations, Reliability and validity, Children

\section{Introduction}

The definition of health as formulated by the World Health Organization, "Health is a state of complete physical, mental and social well-being and not merely the absence of disease or infirmity" is widely accepted nowadays. As the trend is toward chronic rather than acute disease incidence, the effects of healthcare should be measured using the more comprehensive concept of health-related quality of life rather than in terms of mortality or morbidity (Bunge et al., 2005; Matza, Swensen, Flood, Secnik, \& Leidy, 2004). However, because the concept of "quality of life" is abstract and complicated, its attributes cannot be easily identified or measured (Chang \& Oh, 2008). For children, health-related quality of life has a considerable impact on a child's physical and psychological health

Corresponding author Hyun-Ju Kang Department of Nursing, Bucheon University, 25 Shinheung-ro 56beon-gil, Wonmi-gu, Bucheon 420-735, Korea

TEL +82-32-610-0837 FAX+82-32-610-0839 E-MAIL kanghjabc.ac.kr

*This work was supported by the New Faculty Fund provided by Seoul National University in 2010.

Received 6 January 2015 Accepted 26 January 2015

() This is an Open Access article distributed under the terms of the Creative Commons Attribution NonCommercial License (http://creativecommons.org/licenses/by-nc/3.0/) which permits unrestricted noncommercial use, distribution, and reproduction in any medium, provided the original work is properly cited. and development (Kim, Park, Ma, \& Ham, 2013; Page, Thomson, Ukra, \& Baker, 2013; Tezcan \& Simsek, 2013). Therefore, it is necessary to accurately measure a child's health-related quality of life for the child's physical and psychological health and development.

For this, a verified tool that appropriately measures a child's health-related quality of life is required (Grange, Bekker, Noyes, \& Langley, 2007). Although diverse tools are available for measuring quality of life, the target persons are usually adults and elders. Thus, tools for children are not well known, and the tool to measure a child's quality of life that is used outside Korea is mostly for school-aged children and adolescents. Therefore, research on the quality of life of infants or preschool children, who have a more limited ability to express themselves in comparison with adolescents or school-aged children, remains limited. In Korea, thus far, no domestic tool to measure a child's quality of life has been developed, and the tool to measure an adolescent's quality of life is not adequate. In addition, no tool for preschool children has been translated to Korean with the consent of the tool developer as per international standards and with verified reliability and validity.

First developed in the Netherlands and widely used in many European countries and introduced to some of the Asian countries, the Nether- 
lands Organization for Applied Scientific Research Academic Medical Centre TAPQOL tool is applicable to all children, including healthy children and children with diverse chronic diseases. As a parent's proxyreport tool to measure the quality of life of infants over 18 months and preschool children; it includes a balance of physical, social, cognitive, and emotional functions, which are presented as the attributes of quality of life; and its reliability and validity are rated to be appropriate (Fekkes et al., 2000). Although it was evaluated to be suitable for China (Lee et al., 2005), the TAPQOL has not yet been introduced to Korea and other Asian regions.

Therefore, this study was done to identify whether the TAPQOL can be used for Koreans, whose culture and language are different from those of Europeans. In this study, we translated this tool into Korean after obtaining the consent from the tool developer, and then verified the reliability and validity of the Korean version.

\section{Methods}

\section{Participants}

The participants in this study were composed of 2 groups, a group of healthy preschool children's mothers and a group of hospitalized children's mothers within the age group of 18 to 60 months. Based on the standard that 5-10 administrations of items are needed to verify the validity of an instrument, 258 targets (i.e., 6 administrations of the 43 items) were considered adequate. However, 310 questionnaires were collected to allow for possible exclusions due to invalid responses. A total of 250 questionnaires were collected from the nurseries and kindergartens, and 60 were obtained from a university children's hospital. Finally, 223 questionnaires were collected from mothers of the healthy group, and 54 were collected from mothers of the hospitalized group, totaling 277 questionnaires for data analysis.

\section{Instruments}

\section{Health-related quality of life (HRQOL) of preschool children}

The HRQOL of preschool children was assessed by the Korean version of the 43-item TAPQOL. The TAPQOL consists of 12 multi-item scales that cover the domains of physical function, social function, cognitive function, and emotional function. The reporting of specific complaints or limitations is scored on a three-point scale (never, occasionally, and often). The 12 scales considered are sleeping, appetite, lung problems, stomach problems, skin problems, motor functioning, problem be- havior, social functioning, communication, positive mood, anxiety, and liveliness. In the items that belong to the physical and cognitive function domains, the frequency of a specific complaint or limitation during the last 3 months was recorded. Each item was encoded into one single score, ranging from 0 to 4 . A score of 4 was given if the child had no problem. A score of 3 was given if the child had a problem but the child felt "fine" during the problem. A score of 2 was given if the child had a problem and felt "not so good." A score of 1 was given if the child had a problem and felt "quite bad." A score of 0 was given if the child had a problem and felt "bad." In items of social and emotional function domains, a score of 2 was given if the child had "never" had a problem. A score of 1 was given if the child "occasionally" had a problem. A score of 0 was given if the child "often" had a problem (Fekkes et al., 2000). For each scale, the scale score was constructed by summing up the item scores. Finally, all scale scores were linearly transformed into a 0-100 scale, where higher scores indicated a better HRQOL. Cronbach's a ranged from .43 to .84 in Fekkes et al. (2000), in this study, Cronbach's a ranged from .46 to .93 and that of the total was .92 .

\section{Socio-demographic characteristics of the participants}

The socio-demographic characteristics of the children considered in this study were age, gender, institution attendance, gestational age, and hospitalization state, and those of the mothers were age, education level, employment status, marital status, and monthly income.

\section{Procedures}

\section{Translation procedures}

After obtaining permission from the tool developer, translation was performed according to the procedure recommended by the tool developer. First, two experts who were fluent in English and Korean translated the tool to Korean. They completed one translated version by checking whether the data coincided. Then, a native speaker of English who understands Korean well translated the tool in the reverse direction; the result of this translation was re-examined by two pediatric nurses. The translated version and the reverse-translated version were sent to the tool developer so as to obtain final permission for use. We performed a preliminary survey of 10 mothers of preschool children to verify whether there was any problem in understanding before using the tool for data collection.

\section{Data collection}

In 2011, data were collected from four nurseries for the healthy group, 
and one children's hospital in Seoul for the hospitalized group. After explaining the purpose and method of study to the mothers of the preschool children who were eligible for this study, we collected data if they agreed to participate. The study was explained at the nurseries by sending the letter to the mothers at their residential address in order to obtain their written consent. At the hospital, the outline of the study was explained to the practical nurse staff, and the research assistant or hospital nurse obtained the written consent form from the participants prior to data collection.

\section{Ethical considerations}

This study was approved by the Institutional Review Board (IRB) at the researchers' institution. Only those mothers who signed the written consent form were contacted by the researchers or hospital ward nurses. The consent form included a brief description of the study, assurance of confidentiality, and the right to refuse to participate in the study.

\section{Data analysis}

The general characteristics of the participants were determined in terms of real numbers and percentages. The collected data were processed by a factor analysis to confirm the construct validity of the tool. Further, for verifying the known-groups method, we analyzed whether there were any differences in the quality of life between the healthy group and the hospitalized group. The reliability of the tool was measured by using Cronbach's alpha coefficient for each scale. The correlation among the scales was confirmed using Pearson's correlation coefficients.

\section{Results}

\section{Socio-demographic characteristics of the participants}

The socio-demographic characteristics of the participants are presented in Table 1. Of the children, 53.4\% were boys, and 53.8\% of the children were $48-60$ months in age. Approximately $70.1 \%$ of the children attended kindergarten; $91.0 \%$ of the children were born as full-term infants after 38 weeks, and $9.0 \%$ of the children were born as preterm infants at less than 37 weeks of gestational age. Among the children, $80.5 \%$ of the children were in the healthy group and $19.5 \%$ of them were in the hospitalized group.

Most of the mothers were 30-39 years old, and the level of education for $84.8 \%$ was a Junior college degree or higher. Most of the mothers were married, and $62.1 \%$ were unemployed outside the home (Table 1).
Table 1. Socio-Demographic Characteristics of the Participants

$(\mathrm{N}=277)$

\begin{tabular}{|c|c|c|}
\hline Characteristic & $\mathrm{n}$ & (\%) \\
\hline \multicolumn{3}{|l|}{ Children's characteristics } \\
\hline \multicolumn{3}{|l|}{ Gender } \\
\hline Male & 148 & (53.4) \\
\hline Female & 129 & (46.6) \\
\hline \multicolumn{3}{|l|}{ Age (months) } \\
\hline $18-23$ & 6 & $(2.2)$ \\
\hline $24-35$ & 33 & (11.9) \\
\hline $36-47$ & 89 & (32.1) \\
\hline $48-60$ & 149 & (53.8) \\
\hline \multicolumn{3}{|l|}{ Institution attending } \\
\hline Nursery & 52 & (18.8) \\
\hline Kindergarten & 194 & (70.1) \\
\hline None & 31 & (11.1) \\
\hline \multicolumn{3}{|l|}{ Gestational age } \\
\hline Full-term & 252 & (91.0) \\
\hline Preterm & 25 & (9.0) \\
\hline \multicolumn{3}{|l|}{ Hospitalization status } \\
\hline Healthy & 223 & (80.5) \\
\hline Hospitalized & 54 & (19.5) \\
\hline \multicolumn{3}{|l|}{ Mothers' characteristics } \\
\hline \multicolumn{3}{|l|}{ Age (years) } \\
\hline $20-29$ & 13 & (4.7) \\
\hline $30-39$ & 251 & (90.6) \\
\hline$\geq 40$ & 13 & (4.7) \\
\hline \multicolumn{3}{|l|}{ Education level } \\
\hline High school & 42 & (15.2) \\
\hline Junior college or higher & 235 & (84.8) \\
\hline \multicolumn{3}{|l|}{ Currently married } \\
\hline Yes & 275 & (99.3) \\
\hline No & 2 & $(0.7)$ \\
\hline \multicolumn{3}{|l|}{ Work status } \\
\hline Full-time job & 86 & (31.1) \\
\hline Part-time job & 19 & (6.8) \\
\hline None & 172 & (62.1) \\
\hline \multicolumn{3}{|c|}{ Monthly income ( 10,000 won) } \\
\hline 100-199 & 20 & $(7.1)$ \\
\hline $200-299$ & 50 & (18.1) \\
\hline$\geq 300$ & 207 & (74.8) \\
\hline
\end{tabular}

\section{Evaluation of construct validity}

A factor analysis was performed to confirm the construct validity of the tool. A principal component analysis was conducted using varimax rotation, and the Kaiser-Meyer-Olkin (KMO) value, which indicates how well the correlation among variables is explained by other variables, was .87. As a KMO value of more than 90 is judged to be very good and that of .80-.89 is judged to be fairly good (Seo, 2010; Song, 2011), the correlation among the variables in this study was well explained by other variables. Verification of the Barlett's sphericity indicates whether the component analysis model is appropriate or not (Seo, 2010; Song, 2011), the probability considered significant in this study was found to be $p<.001$, which is appropriate for a component analysis model. 
Table 2. Summary of Factor Analysis of the TAPQOL

\begin{tabular}{|c|c|c|c|c|c|c|c|c|c|c|c|}
\hline \multirow{2}{*}{ Scale and its items } & \multicolumn{11}{|c|}{ Factor loadings } \\
\hline & 1 & 2 & 3 & 4 & 5 & 6 & 7 & 8 & 9 & 10 & 11 \\
\hline
\end{tabular}

\begin{tabular}{ll}
\hline Factor 1. Positive mood & \\
Item 24.* In good spirits & .90 \\
Item 25. Cheerful & .91 \\
Item 26.* Happy & .87 \\
Item 30.* Energetic & .79 \\
Item 31.* Active & .71 \\
Item 32.* Lively & .78
\end{tabular}

$\begin{array}{ll}\text { Factor 2. Motor functioning } & .87 \\ \text { Item 36. Difficulty with walking } & .87 \\ \text { Item 37. Difficulty with running } & .84 \\ \text { Item 38. Difficulty with walking up stairs without help } & .80\end{array}$

\section{Factor 3. Problem behavior}

Item 17. My child was short-tempered $\quad .69$

Item 18. My child was aggressive $\quad .75$

Item 19. My child was irritable $\quad .62$

Item 20. My child was angry $\quad .71$

Item 21. My child was restless or impatient with me $\quad .74$

Item 22. My child was defiant/awkward with me $\quad .75$

Item 23. I could not manage my child $\quad .65$

Factor 4. Communication

Item 40. Difficulty in understanding what others said

Item 41. Difficulty in talking clearly $\quad .82$

Item 42. Difficulty in saying what he/she meant $\quad .86$

Item 43. Difficulty in making it clear what he/she wanted $\quad .78$

Factor 5. Sleeping

Item 10. Did your child sleep restlessly?

Item 11. Was your child awake at night? $\quad .79$

Item 12. Did your child cry at night? $\quad .78$

Item 13. Did your child have difficulty sleeping through the night? 68

Factor 6. Appetite

Item 14. Was your child's appetite poor?

Item 15. Did your child have difficulty eating enough?

Item 16. Did your child refuse to eat?

Factor 7. Social functioning

Item 33.* My child was able to play happily with other children $\quad .76$

Item 34 * My child was at ease with other children

.83

Item 35.* My child was confident with other children

.76

Factor 8. Anxiety

Item 27. Frightened

Item 28. Tense

Item 29. Anxious

Factor 9. Skin problems

Item 3. Eczema

.63

Item 4. Itchiness

.83

Item 5. Dry skin

.83

Factor 10. Lung problems

Item 6. Bronchitis

Item 7. Difficulty with breathing or lung problems

50

.76

.56

Item 8. Shortness of breath

Factor 11. Stomach problems

Item 1. Stomach-ache or abdominal pain $\quad .42$

Item 2. Colic

.87

Item 9. Nauseous 
Table 2. Continued

\begin{tabular}{|c|c|c|c|c|c|c|c|c|c|c|c|}
\hline \multirow{2}{*}{ Scale and its items } & \multicolumn{11}{|c|}{ Factor loadings } \\
\hline & 1 & 2 & 3 & 4 & 5 & 6 & 7 & 8 & 9 & 10 & 11 \\
\hline Eigenvalue & 11.31 & 4.55 & 3.25 & 2.34 & 1.88 & 1.72 & 1.56 & 1.45 & 1.37 & 1.09 & 1.07 \\
\hline Cumulative \% & 26.30 & 36.89 & 44.44 & 49.88 & 54.26 & 58.26 & 61.87 & 65.24 & 68.43 & 70.97 & 73.45 \\
\hline
\end{tabular}

* Reverse-scored items.

TAPQOL = TNO-AZL Preschool Children Quality of Life.

Table 3. Comparison of TAPQOL Scores between the Hospitalized Group and the Healthy Group

\begin{tabular}{|c|c|c|c|c|c|c|}
\hline \multirow{2}{*}{ Domain and scale } & \multicolumn{2}{|c|}{ Hospitalized group $(n=54)$} & \multicolumn{2}{|c|}{ Healthy group $(n=223)$} & \multirow{2}{*}{$\mathrm{t}$} & \multirow{2}{*}{$p$ value } \\
\hline & Mean & SD & Mean & SD & & \\
\hline \multicolumn{7}{|l|}{ Physical function } \\
\hline Sleeping & 70.49 & 19.33 & 87.22 & 14.49 & 5.97 & .001 \\
\hline Appetite & 73.31 & 23.19 & 83.64 & 19.38 & 3.38 & .001 \\
\hline Lung problems & 81.49 & 20.70 & 93.24 & 11.46 & 4.02 & .001 \\
\hline Stomach problems & 87.81 & 16.95 & 94.96 & 9.00 & 3.00 & .004 \\
\hline Skin problems & 84.88 & 18.13 & 89.55 & 15.42 & 1.31 & .191 \\
\hline Motor functioning & 85.76 & 22.82 & 97.81 & 8.94 & 3.81 & .001 \\
\hline \multicolumn{7}{|l|}{ Social function } \\
\hline Problem behavior & 67.21 & 21.47 & 72.11 & 19.74 & 1.61 & .109 \\
\hline Social functioning & 73.46 & 34.79 & 88.87 & 18.36 & 3.15 & .003 \\
\hline \multicolumn{7}{|l|}{ Cognitive function } \\
\hline Communication & 78.70 & 27.05 & 93.11 & 12.21 & 3.82 & .001 \\
\hline \multicolumn{7}{|l|}{ Emotional function } \\
\hline Positive mood & 175.93 & 40.52 & 185.88 & 33.94 & 1.67 & .100 \\
\hline Anxiety & 62.04 & 22.76 & 77.81 & 21.67 & 4.75 & .001 \\
\hline
\end{tabular}

TAPQOL = TNO-AZL Preschool Children Quality of Life.

From the results of the principal component analysis with varimax rotation, 11 factors with an eigenvalue of more than 1 were extracted. As the factor loading value of all items was more than .4, no item was excluded. Usually, a factor loading value of more than .4 is used as a standard for a component analysis (Yang, 1998). The percentage of the explained variance by factor was $26.30 \%$ for factor $1,10.59 \%$ for factor 2 , $7.55 \%$ for factor $3,5.44 \%$ for factor $4,4.38 \%$ for factor $5,4.00 \%$ for factor 6 , $3.61 \%$ for factor $7,3.37 \%$ for factor $8,3.19 \%$ for factor $9,2.53 \%$ for factor 10 , and $2.48 \%$ for factor 11 . The cumulative explained variance was 73.45 $\%$ (Table 2).

The tools of Fekkes et al. (2000) were composed of 12 scales, but 11 factors were extracted using the factor analysis in this study because the two scales of "Positive mood" and "Liveliness" in the original tool were extracted in one bundle of factors. The items describing "Positive mood" included "in good spirits," "cheerful," "happy," "energetic," “active," and "lively." Thus, the factor was named "Positive mood." Other factors were named using the same terms used in the original tool of Fekkes et al. (2000). They were factor 1: positive mood, factor 2: motor functioning,
Table 4. Internal Consistency of the TAPQOL Questionnaire

$(\mathrm{N}=277)$

\begin{tabular}{lcc}
\hline Domain and its subscales & Number of items & Cronbach's alpha \\
\hline Sleeping & 4 & .87 \\
Appetite & 3 & .90 \\
Lung problems & 3 & .59 \\
Stomach problems & 3 & .46 \\
Skin problems & 3 & .71 \\
Motor functioning & 4 & .95 \\
Problem behavior & 7 & .84 \\
Social functioning & 3 & .89 \\
Communication & 4 & .93 \\
Positive mood & 6 & .93 \\
Anxiety & 3 & .78 \\
Total & 43 & .92 \\
\hline
\end{tabular}

TAPQOL = TNO-AZL Preschool Children Quality of Life.

factor 3:problem behavior, factor 4: communication, factor 5: sleeping, factor 6: appetite, factor 7: social functioning, factor 8: anxiety, factor 9: skin problems, factor 10: lungs problems, and factor 11: stomach problems (Table 2). 
Table 5. Pearson Correlation Coefficients among the 11 Scales of the TAPQOL

\begin{tabular}{|c|c|c|c|c|c|c|c|c|c|c|}
\hline Scale & 1 & 2 & 3 & 4 & 5 & 6 & 7 & 8 & 9 & 10 \\
\hline \multicolumn{11}{|l|}{ 1. Positive mood } \\
\hline 2. Motor functioning & $0.36^{* *}$ & & & & & & & & & \\
\hline 3. Problem behavior & 0.12 & 0.02 & & & & & & & & \\
\hline 4. Communication & $0.36^{* *}$ & $0.63^{* *}$ & $0.15^{*}$ & & & & & & & \\
\hline 5. Sleeping & $0.30^{* *}$ & $0.38^{* *}$ & $0.33^{* *}$ & $0.40^{* *}$ & & & & & & \\
\hline 6. Appetite & $0.30^{* *}$ & $0.24^{*}$ & $0.35^{* *}$ & $0.34^{* *}$ & $0.47^{* *}$ & & & & & \\
\hline 7. Social functioning & $0.52^{* *}$ & $0.45^{* *}$ & $0.13^{*}$ & $0.49^{* *}$ & $0.34^{* *}$ & $0.33^{* *}$ & & & & \\
\hline 8. Anxiety & $0.13^{*}$ & $0.19^{* *}$ & $0.19^{* *}$ & $0.27^{* *}$ & $0.34^{* *}$ & $0.37^{* *}$ & $0.24^{* *}$ & & & \\
\hline 9. Skin problem & 0.05 & 0.04 & $0.16^{* *}$ & $0.14^{*}$ & $0.22^{* *}$ & $0.17^{* *}$ & $0.13^{*}$ & $0.20^{* *}$ & & \\
\hline 10. Lung problem & $0.28^{* *}$ & $0.40^{* *}$ & $0.23^{* *}$ & $0.34^{* *}$ & $0.43^{* *}$ & $0.35^{* *}$ & $0.33^{* *}$ & $0.29^{* *}$ & $0.30^{* *}$ & \\
\hline 11. Stomach problem & $0.19^{* *}$ & $0.22^{* *}$ & $0.28^{* *}$ & $0.29 * *$ & $0.34^{* *}$ & $0.41^{* *}$ & $0.22^{* *}$ & $0.34^{* *}$ & $0.32^{* *}$ & $0.42^{* *}$ \\
\hline
\end{tabular}

${ }^{*} p<.05 ;{ }^{* *} p<.01$.

TAPQOL = TNO-AZL Preschool Children Quality of Life.

\section{Evaluation of the validity of the known-groups method}

In the case of all scales except skin problems $(p=.191)$, and problem behavior $(p=.109)$, and positive $\operatorname{mood}(p=.100)$, the hospitalized group exhibited significantly lower mean TAPQOL scores than the healthy group (Table 3)

\section{Reliability}

All scales except stomach and lung problems in the physical function domain exhibited Cronbach's alpha coefficients greater than .70 (Table 4).

\section{Correlation among 11 scales}

Analyzing the correlation among the 11 scales of the TAPQOL, we found that most of scales exhibited a statistically significant correlation. The highest correlations was between motor functioning and communication $(\mathrm{r}=.63)($ Table 5).

\section{Discussion}

This study was performed to verify the reliability and validity of the TAPQOL for measuring health-related quality of life of Korean children who are in the age group of 18 months to 5 years. First, for the 11 scales, the internal consistency was good in general (Cronbach's a ranged from .59 to .93 and was more than .70 for most of the scales). The result of exceptionally low reliability of the stomach problem scale, as obtained in this study, coincides with the findings of Lee et al. (2005) and Fekkes et al. (2000). There is a study that reported that some respondents had some confusion about the meaning of stomachache and colic during the interview process. Further, the original study of TAPQOL concluded that the cause of the low reliability of the scale was the low prevalence and variance of the problem in the general population sample for the scale (Fekkes et al., 2000). In fact, the researchers were told that the mothers of this study said that it was difficult for them to differentiate between "stomachache or abdominal pain" and "colic" (the items included in the stomach problem scale). Also, they said that it was difficult to understand "bronchitis", "lung problem", and "shortness of breath", term in the scale of lung problems. Because the mothers were unfamiliar with these terms, they found it difficult to correctly answer the relevant questions. Thus, in the Korean questionnaires in a future study, it may be necessary to change the terms to more commonly used alternatives that can be understood more easily by mothers.

According to the factor analysis, the total 12 scales of the original tool were categorized as 11 scales in this study. Most of the items from the original Dutch version were included in the Korean version. However, the positive mood and liveliness scales in the original version were united into one scale called the positive mood scale. This result is similar to that of another study that tested the validity of the Spanish version of the TAPQOL (Rajmil et al., 2011). In that study, the "happy" item in the positive mood scale and the "frightened" item in the anxiety scale were classified into another scale. Although the TAPQOL measures diverse aspects of children's health-related quality of life, the positive mood, liveliness, and anxiety scales in the case of emotion-based quality of life have been expressed in different ways in each country evaluated in the past. These differences have been attributed to different cultures and emotions of the countries. While Korean mothers consider all of the emotions such as "energetic," "active," and "lively" in the scale of liveliness to be without differentiation from those such as "in good spirits," 
"cheerful," and "happy" in the scale of positive mood, the mothers in European countries like Spain and the Netherlands interpreted emotions by subdividing them into positive mood, liveliness, and anxiety scales. To confirm this interpretation, a replication study may be necessary to identify whether the results of the study for various cultural backgrounds are consistent with this result. Also, further studies using factor analysis and item response theory in large samples of children under the age of 5 years would permit identification of items that function differently across groups such as age, culture, or other social backgrounds (Rajmil et al., 2011)

In this study, the Pearson correlation coefficients that were statistically significant among the 11 scales were found to be from .13 to .63 , which is not very high. This coincides with the results of Fekkes et al. (2000), who developed the TAPQOL tool, and of the studies that validated the TAPQOL in China (Lee et al., 2005) and Taiwan (Chien, Chou, Ko, \& Lee, 2006), showing that the TAPQOL tool measures diverse aspects of children's health-related quality of life and that the scales do not overlap (Fekkes et al., 2000). Although the TAPQOL in this study was composed of 11 scales, which was different from the original version, it is a valid tool that can measure Korean children's health-related quality of life.

To verify the validity by comparing the known-groups using the TAPQOL, we compared whether there were differences in the TAPQOL between the healthy and the hospitalized groups. For the measurement of children's health-related quality of life, the participants are usually divided into a healthy group and an unhealthy group such as children who are chronically ill, have cancer, or were preterm (Fekkes et al., 2000; Grange et al., 2007). In this study, we compared healthy children and hospitalized children because hospitalization may be a primary factor that considerably affects children's health-related quality of life. As a result, the score of the quality of life of the hospitalized group was significantly low in the case of most of the scales, thereby verifying the validity of the contrasted group. In other studies, the score of the quality of life of the hospitalized group was significantly lower than the healthy group, which coincided with the result of this study (Bont, Steijn, van Aalderen, \& Kimpen, 2004; Rajmil et al., 2011). Based on this result, it may be necessary to construct a system that can constantly monitor and control hospitalized children's quality of life. Further, to evaluate the effect of various kind of care including nursing intervention for hospitalized children it is meaningful to assess changes in their quality of life by using the TAPQOL tool.

Recently, efforts have been made to measure children's health-related quality of life by using several tools. However, the Health Utilities Index (HUI), one of the most widely used measurements in pediatric studies, has been applied only to special groups such as cancer care unit or pediatric intensive care unit patients. Moreover, its content validity is weak because it does not cover all dimensions of the health-related quality of life. In addition, it was developed only for children over 5 years old, mainly in Canada (Grange et al., 2007). The Pediatric Quality of Life Inventory (PedsQL) tool has also been well researched. It was developed from earlier measures for children with cancer specifically (Varni et al., 1998). The recent version of PedsQL was revised to be age appropriate, and researchers attempted to validate it for children less than 2 years old (Varni et al., 2011). However, thus far, it has not yet been validated for children from various cultural backgrounds.

Therefore, the TAPQOL questionnaire that considers four domains of health-related quality of life for 1-5-year-old children can be useful to assess the quality of life for infants and preschoolers under various conditions. The TAPQOL has been acceptable for discriminating between healthy and unhealthy children in countries where languages other than English are spoken (Lee et al., 2005; Rajmil et al., 2011). It is based on the parent's perception of his or her child's health-related quality of life because self-reports are impossible to obtain from preschool children. Although its reliability is controversial, it is known that the response of parents is generally reliable (Matza et al., 2004).

The presented results need to be interpreted with caution, due to some limitations of the study. First, this study did not perform confirmatory factor analysis process to further ensure the validity of the tool. Thus, we propose to try confirmatory factor analysis study to increase the validity of the tool. Second, in this study a convenience sample was used. Also, the difference in sample size between the healthy and hospitalized group might affect the result of this study. The results of this study might not be generalized to preschool children and mothers in other countries. This study was applied for the first time in Korea. Therefore, in the future there may be a need to re-check the results by repeating the study.

In summary, this study showed that the Korean version of TAPQOL is a valid and reliable tool for preschool children in Korea. Therefore, it is expected to be utilized as a tool that can measure diverse aspects of children's health-related quality of life in clinical and community settings. In future research, it would be meaningful to compare the health-related quality of life of preschool children among countries by using the TAPQOL. 


\section{Conclusion}

As the reliability and validity of the Korean version of the TAPQOL tool that measures children's health-related quality of life are verified, this tool is deemed to be appropriate for measuring children's health-related quality of life in diverse clinics and community settings in Korea. In particular, it may be utilized as a tool to measure the health-related quality of life of children with chronic diseases and hospitalization whose health-related quality of life may be negatively affected.

\section{Conflict of Interest}

No potential conflict of interest relevant to this article was reported.

\section{Acknowledgements}

This work was supported by the New Faculty Fund provided by Seoul National University in 2010.

\section{References}

Bont, L., Steijn, M., van Aalderen, W. M., \& Kimpen, J. L. (2004). Impact of wheezing after respiratory syncytial virus infection on health-related quality of life. The Pediatric Infectious Disease Journal, 23(5), 414-417.

Bunge, E. M., Essink-Bot, M. L., Kobussen, M. P., van Suijlekom-Smit, L.

W., Moll, H. A., \& Raat, H. (2005). Reliability and validity of health status measurement by the TAPQOL. Archives of Disease in Childhood, 90(4), 351-358. http://dx.doi.org/10.1136/adc.2003.048645

Chang, E. Y., \& Oh, J. A. (2008). A concept analysis of the quality of life for the children. Korean Parent-Child Health Journal, 11, 127-137.

Chien, L. Y., Chou , Y. H., Ko, Y. L., \& Lee, C. F. (2006). Health-related quality of life among 3-4-year-old children born with very low birthweight. Journal of Advanced Nursing, 56(1), 9-16.

Fekkes, M., Theunissen, N. C. M., Brugman, E., Veen, S., Verrips, E. G. H., Koopman H. M., et al. (2000). Development and psychometric evaluation of the TAPQOL: A health-related quality of life instrument for 1-5-year-old children. Quality of Life, 9(8), 961-972.

Grange, A., Bekker, H., Noyes, J., \& Langley, P. (2007). Adequacy of healthrelated quality of life measures in children under 5 years old: Systematic review. Journal of Advanced Nursing, 59(3), 197-220. http://dx.doi. org/10.1111/j.1365-2648.2007.04333.x

Kim, H. S., Park, J. Y., Ma, Y. M., \& Ham, O. K. (2013). Factors influencing health-related quality of life of overweight and obese children in south Korea. The Journal of School Nursing, 29, 361-369.

Lee, C., Chien, L., Koc, Y., Choud, Y., Taie, C., \& Lioua, Y. (2005). Development and psychometric properties of the Chinese language version of the TAPQOL: A health-related quality of life instrument for preschool children. International Journal of Nursing Studies, 42(4), 457-465.

Matza, L. S., Swensen, A. R., Flood, E. M., Secnik, K., \& Leidy, N. K. (2004). Assessment of health-related quality of life in children: A review of conceptual, methodological, and regulatory issues. Value in Health, 7(1), 79-92. http://dx.doi.org/10.1111/j.1524-4733.2004.71273.x

Page, L. A., Thomson, W. M., Ukra, A., \& Baker, S. R. (2013). Clinical status in adolescents: Is its impact on oral health related quality of life influenced by psychological characteristics? European Journal of Oral Sciences, 121(3), 182-187. http://dx.doi.org/10.1111/eos.12034

Rajmil, L., Abad, S., Sardon, O., Morera, G., Pe'rez-Yarza, E. G., Moreno, A., et al. (2011). Reliability and validity of the Spanish version of the TAPQOL: A health-related quality of life (HRQOL) instrument for 1to 5-year-old children. International Journal of Nursing Studies, 48(5), 549-556. http://dx.doi.org/10.1016/j.ijnurstu.2010.09.004

Seo, U. (2010). Statistical analysis using the SPSS 18.0 program. Gyeonggi province: Freedom Academy.

Song, J. (2011). Statistical analysis methods. Gyeonggi province: 21st Century.

Tezcan, S., \& Simsek, T. T. (2013). Comparison of health related quality of life between children with cerebral palsy and spine bifida. Research in Developmental Disabilities, 134(9), 2725-2733. http://dx.doi.org/10.1016/ j.ridd.2013.05.017

Varni, J. W., Katz, E. R., Seid, M., Quiggins, D. J., Friedman-Bender, A., \& Castro, C. M. (1998). The Pediatric Cancer Quality of Life Inventory (PCQL). I. Instrument development, descriptive statistics, and crossinformant variance. Journal of Behavioral Medicine, 21(2), 179-204.

Varni, J. W., Limbers, C. A., Neighbors, K., Schulz, K., Lieu, J. E., Heffer, R.W., et al. (2011). The PedsQL Infant Scales: Feasibility, internal consistency reliability, and validity in healthy and ill infants. Quality of Life Research, 20(1), 45-55. http://dx.doi.org/10.1007/s11136-010-9730-5

Yang B. Understanding and application of multivariate data analysis. Seoul: Hak Jisa. 